\title{
Promotion of Perinatal Mental Health
}

\author{
Bhabani Adhikari \\ Lecturer, Sahid Smiriti Multiple Campus, Chitwan \\ Email for correspondence: bhabaniadhikari0@gmail.com
}

\begin{abstract}
The aim of this paper is to discuss different aspects of perinatal mental health like; risk factor, impact, issues and to suggest the guidelines for promotion of perinatal mental health. Perinatal mental health is an emerging concept, generally refers to the period 22 completed weeks (i.e. 154 days) of gestation and ending seven completed days after birth. During the pregnancy women may suffer from different mental health problems such as anxiety and depression. Lack of nutritious food, proper healthy housing, and adequate time for rest, quality health services and positive support by the intimacy partner are major responsible factors for getting mental disorder during perinatal period. Problem with in-laws and domestic violence are also responsible for this. Individual, family, community and national agencies should aware the problem and difficulties of the perinatal period and ways to eliminating the associated problems by their action and efforts. Without waiting the sign and symptoms of depression all well pregnant women need to be screened as part of their perinatal health check.
\end{abstract}

Key words: Mental health, depression, perinatal depression, pregnancy and maternal health.

\section{Introduction}

As perinatal mental health and perinatal mental illness are emergent concepts, international consensus has not yet been reached on key issues such as diagnostic criteria and temporality (Paykel, 2002). For women of reproductive age, the biological functions of pregnancy and childbirth create additional health needs (United Nations [UN], 2015). Indeed, agreed definitions and diagnostic criteria do not currently exist for postnatal/postpartum depression, despite the condition having been the subject of much academic and media focus in recent decades. The 10th revision of the International Statistical Classification of Diseases and Related Health Problems (ICD-10) defines the perinatal period as commencing at 22 completed weeks or 154 days of gestation and ending seven completed days after birth (World Health Organization [WHO], 1992). While some academics and clinicians suggest that the perinatal period and associated illnesses might be extended into the early years after birth (Najman, Andreson, Bor, Callaghan \& Williams, 2000). Perinatal mental disorders (PMDs) are the most common complication of pregnancy and the first postpartum year (Bateson, Karen, Darwin, Josephine, Galdas \& Michel, 2017). No matter low or high income of the countries pregnant mother's mental health is equally important for both of them. During the pregnancy women may suffer from different mental health problems such as anxiety and depression. The physical impacts of pregnancy are widely discussed and 
recounted by most of the women. In comparison, the emotional changes during this time attract less attention and are less well publicized (Boots Family Trust [BFT], 2013).

Emotional changes are common during pregnancy and in the first weeks following the birth of a baby. Factors, such as rape, HIV/AIDS, adolescent pregnancy, refugee status and substance use, are linked with poor maternal mental health. Nepal has the perinatal mortality rate is 31 deaths per 1,000 pregnancies (Nepal Demographic Health Survey [NDHS], 2016). There are many stressful events that can arise at different stages of pregnancy and have a negative and positive effect on a mother's emotional health (Flisher, 2013). In the perinatal period different kind of problems were experienced by the mothers such as feelings of despair, panic, inability to cope, and even suicidal thoughts and of putting her baby up for adoption (Burgess, 2011). Impact of mothers experience on the baby can last for a long time. Even when the mother's mood improves the outcome for her child may not. Babies of depressed mothers tend to be more 'fussy' (that is, they cannot settle or be soothed and may cry for long periods, even though they are healthy and their basic needs are met). They also tend to be less responsive to caretakers' facial and vocal expressions, more inactive and have elevated stress hormones compared to infants of non-depressed mothers (Goodman \& Gotlib, 2002). Early detection of any mental health problems can help prevent future serious psychological disorders. Not only women with symptoms of depression need assessment and evaluation of psychological problems, but also all well women need to be screened as part of their perinatal health check (Khajehei, 2015).

\section{Research Questions}

- What are the impacts of perinatal mental disorder?

- What are the issues and challenges of perinatal mental health?

- How perinatal mental health can be promoted?

\section{Materials and Methods}

The study applied descriptive method for appraising secondary nature of information. For that purpose, various international and national literatures related to perinatal mental health issues were reviewed and appraised. Terms searched were perinatal mental health, mental health, perinatal disorders, common mental disorders, postnatal depression, perinatal health, pre-natal depression. Among 1,125 full-text papers, extracted data, and independently appraised. Hence, this paper is developed on the basis of different literatures like books, reports and peer reviewed journals. During appraisal, focus was given to explore major issues and challenges of perinatal mental disorder as well as corrective measures for promoting such issues and challenges.

\section{Risk Factors for Perinatal Mental Disorder}

Perinatal mental disorders are among the commonest health problems associated with pregnancy and the postpartum period. Antenatal disorders (including depression and anxiety disorders, eating disorders, and psychoses) are associated with adverse effects on the fetus including low birth weight and pre-term delivery. In order to promote the perinatal mental health, first of all we have to find out the major causes which lead to pregnant women in mental disorder situation. A systematic review study suggested domestic violence in the perinatal period is associated with adverse obstetric outcomes, but evidence is limited on its association with perinatal mental disorders. High levels of symptoms of all types of perinatal mental disorders included in studies (i. e., antenatal and postnatal 
anxiety, depression) were associated with having experienced domestic violence (Howard, Oram, Galley, Treyillion, \& Feder, 2013). Pregnancy and the postnatal period is a psychologically distressing time for many women, particularly those living in poverty, or with violence, abuse or HIV/AIDS. The burden of maternal mental illness (e.g. mental illness during and after pregnancy) in low and middleincome countries is high (Flisher, 2013).

Because of the low economic status it is very difficult to get adequate nutritious food, proper healthy housing, and adequate time for rest and quality health services. Besides these different kind of abuses (e.g. physical, sexual, verbal, emotional, psychological and economic abuses) and harassment may be the cause of mental disorder or mental distress or mental illness. The psychological wellbeing of fathers can have a direct impact on maternal mental health, both positively and negatively. Having a supportive partner is a protective factor against developing mental health problems (Lancester et al., 2010, Bateson et al., 2017). If there is no good relation between partners perinatal mental health problems occur frequently. A father can contribute significantly to mother and baby's well-being, even under the most difficult circumstances, and if his support is not forthcoming this represents a significant deficit for the family (Oakes, 2003). A previous history of mental health problem, such as depression or anxiety, has been reported to be a strong predictor of perinatal mental illnesses. Half the women with prenatal depression will continue to feel depressed throughout pregnancy and during postnatal period (Khajahei, 2015). All in all, unplanned pregnancy, high risk pregnancy, young age, being unmarried, intimate partner violence and interpersonal issues with in-laws are also the risk factors of perinatal mental illness.

\section{Impact of Perinatal Mental Disorder}

Perinatal mental illnesses negatively affect women's interpersonal relationship and quality of life and have in/direct negative impacts on short-term and long-term physical and mental health of their children. Mental stress and anxiety have been found to be associated with significant risks to the baby, including preterm labour and low birth weight and behavioural and emotional problems in the child (Weinstock, 2008 as cited in Barber, 2009). Another study argued that family trust, more than a quarter (28\%) reported that they had trouble bonding with their baby and more than one in five $(22 \%)$ reported having suicidal thoughts as well as ntrusive thoughts and self-harm were also identified (Howard, Oram, Galley, Treyillion \& Feder, 2013). Meta analysis of data from longitudinal studies suggests that women who experience domestic violence during pregnancy have three fold increase in unadjusted odds of probable depression in the postnatal period. There is growing evidence that, in low/middle income countries, the negative effects of maternal mental disorders on the growth and development of infants/children are independent of the influence of poverty, malnutrition and chronic social adversity (Stewart, 2007).

\section{Issues and Challenges of Perinatal Mental Health}

A review of literature on perinatal mental health reflects a growing sentiment in the scientific community that having no information regarding the impact of psychiatric treatment on the mother and developing fetus/infant poses dangers that may exceed the risks involved in research (Brandon, Geetha, Lee, Inring \& Sadler, 2009). The maternal mortality ratio (maternal deaths) in Nepal has gradually declined over the past two decades; however, it is still very high in remote rural pockets. Grassrootslevel health workers are not well trained to deal with mental health issues. This made us think about 
the training needs of health care workers serving pregnant women, or indeed women of child-bearing age. The causes of perinatal mortality and mental ill health have significantly shifted over time; hence essential to change the mental health training to reflect today's societal need (Teijlingen, Simkhada, Devkota, Fanning, Ireland, Simkhada, Sherchan, Silwal, Pradhan, S.K, Maharjan \& R.K. Maharjan, 2015).

Pregnant women have a domestic duty but they are dependent on family members to provide for and protect them, and they lack agency concerning decisions about healthcare, fertility, nutrition, their children's education and health, and movements outside the home. Lack of self decision making power about their health is also a challenging the promotion of perinatal health (Clark et al., 2014). Distressed mothers discussed several strategies to alleviate tension, including seeking treatment for perceived physical health problems and tension from doctors or dhamis, having repeated pregnancies until a son was delivered, manipulating social circumstances in the household, and deciding to accept their fate(ibid). Governmental mental health research body also has a strong role in promotion of perinatal mental health, lack of structure or exclusion of mental health from health research mechanism is another challenge for promotion of perinatal mental health (Yasami et al., 2011). Early marriage and giving preference for the son are also inviting perinatal mental health disorder in relation to south Asian countries.

\section{Promotion of Perinatal Mental Health}

Maternal health status of each mother depends up on the available facilities of the maternal health care in their access. Mainly antenatal, intra natal and post natal cares comes under the maternal health care package which prevents pregnancy related death and other complications (Bhandari, 2012). Psychological and educational components of the interventions must be adapted to the circumstances in which women in low and middle income countries live. In places where women live in densely populated communities and crowded households, involving the entire family and community in their care tends to be more beneficial than an individualistic approach (Rahman et al., 2013). All women should be asked early in their pregnancy about a previous history of serious psychiatric disorder and that management plans should be in place with regard to the high risk of recurrence following delivery (Oakes, 2003). All women require admission to a psychiatric unit in the late pregnancy or the postpartum period should be admitted together with their infant to a specialized mother and baby unit. The personnel who involved in the care of pregnant or post partum women should have training in the normal emotional changes associated with pregnancy and the post partum period, the maternity context, psychological distress, perinatal disorder and parent child relationship issues. Women should have access to psychological and psychosocial treatments. A good parent infant mental health service should be provide care for mothers with complex perinatal mental health problems who have or are at risk of parenting difficulties

The activities of mental health promotion are mainly socio-political: reducing unemployment, improving schooling and housing, working to reduce stigma and discrimination of various types. The key agents are politicians, educators, and members of nongovernment organizations (WHO, 2004, p. 26). Recent study indicates that skilled attendance at every birth; safe blood supplies, staff training, building management capacity, improving monitoring systems and use of process indicators, promoting dialogue between women and providers on quality of care, and increasing equity and access at district level are most important area of maternal health promotion (Baker, Bird, Pradhan \& Shakya, 
2007). To address financial barriers to women accessing maternity services, which was initiated by the Nepal government has initiated Maternity incentive scheme in 75 districts in August 2005. It creates an incentive to deliver in a health facility by covering transport costs for all pregnant women and providing free services for women (Baker et al., 2007). Such kind of incentive program will be very helpful to promote the perinatal mental health of poor women because it helps reduce the anxiety of rent to rich the hospital.

\section{Guidelines for Perinatal Mental Health Promotion}

(WHO, 2000) developed some normative guidelines for reducing the maternal and perinatal mortality and morbidity as follows:

- Establish (or update) national policy and standards for family planning, induced abortion (where it is not against the law), maternal and newborn care (including post-abortion care);

- Develop a combination of regulatory measures;

- Develop systems for ensuring that these standards are properly implemented;

- Improve access to cost-effective maternal and newborn care and fertility regulation services through promoting increased investments in the public sector and arrangements (such as contracting) to maximize the contribution of the private health sector to the national health goals;

- Encourage and foster practices, at home, in families and in communities, that promote maternal and newborn health, and fertility regulation;

- Improve systems for monitoring maternal and newborn health and care services, including fertility regulation services; and keep safe motherhood high on national health and development agendas. (Portela \& Santarelli, 2003, pp 60-62), suggested following four priority areas for promotion of maternal and newborn health:

- Developing capacities of women, families and communities to stay healthy, make healthy decisions and respond to obstetric and neonatal emergencies;

- Increasing awareness of women, families and communities of their sexualand reproductive rights, and of the needs and potential problems related to maternal and newborn health;

- Strengthening linkages for social support between women, men, families and communities and with the health care delivery system;

- Improving quality of care, health services and health provider interactions with women, men, families and communities. (Bhandari, 2012, p.68), suggested some ways to promote maternal health as follows:

- Improve access to increase utilization of health services;

- Make maternal service affordable at all level for creating demand;

- Produce trained skilled human resources and expansion/decentralization of health facilities;

- Intervention against socio-cultural practice and involvement of male in maternal health care;

- Improve women status in house hold and society;

- Establish communication network and develop awareness on maternal health care;

\section{Conclusion}

Empowering together of women, families and communities is necessary for promotion of perinatal mental health. Women who experience domestic violence during pregnancy have a big chance of depression in the post natal period. Husband's supportive role is extremely important to reducing 
the chance of depression during perinatal period. If mother has experienced mental disorder during perinatal period her child also may be affected by different kind of health problems. Family, community, government and non-government organizations all have a great responsibility to promote the health of pregnant women during perinatal period to develop the peaceful and healthy society. To promote the perinatal maternal health it is necessary to support in some key areas like policy development and planning, service strengthening, infrastructure development, technical and managerial improvements, development of skilled human resource, particularly for skilled birth attendants, increasing equity and access, financing demand creation, and monitoring and health information dissemination.

\section{References}

Baker, C., Bird, E., Pradhan, A. \& Shakya, G. (2007) Support to the Safe Motherhood Programme in Nepal: An Integrated Approach. Reproductive Health Matters, 15 (30), 81-90. DOI: http://dx.doi.org/10.1016/S0968-8080 (07)30331-5.

Barber, C. C. (2009). Perinatal Mental Health Care in New Zealand: The Promise of Beginnings. New Zealand Journal of Psychology, 38 (1),

Bateson, K., Josephine, D., Galdas Z. \& Michel, P. (2017). Engaging Fathers: Acknowledging the Barriers. Journal of Health Visiting, 122-128. Retrieved from http://eprints.whiterose. ac.uk/114122/

Boots Family Trust (BFT). (2013). Perinatal Mentnal Health. Experience of Women and Health Professionals. Registered charity no: 1154023, Designed by Soapbox, www.soapbox.co.uk

Bhandari, T. R. (2012). Maternal Health issues of Nepal and ways Forward. JHAS, 2 (1), 64-69

Brandon, A. R., Geetha, L S. C., Inring, S. J. \& Sadler, J. Z. (2009). Ethical Issues in Perinatal Mental Health Research. Curr Opin Psychiatry, 22 (6), 601-606.

Doi:http://dx.doi.org/10.1097/YCO.0b013e3283318e6f

Clark et al., (2014). Understanding psychological distress among mothers in rural Nepal: a qualitative grounded theory exploration. BMC Psychiatry.

DOI: http://10.1093/bmb/ldg011

Flisher, A. J. (2013). Maternal Mental Health .A handbook for health workers. ISBN (ebook) 978-0620-56428-1 www.pmhp.za.org

Howard, L. M., Oram, S. ,Galley, H.,Treyillion, K. \& Feder, G. (2013). Domestic Violence and Perinatal Mental Disorders: A Systematic Review and Meta-Analysis. Retrieved from http://dx.doi.org/10.1016/S0968-8080(07)30331-5

Khajehei, M. (2015). Mental Health of Perinatal Women. World Journal of Obstetrics and Gynocology, $4(2)$.

DOI: http://10.5317.

Najman, J. M., Andreson, M. J., Bor, W., Callaghan, M. J. O \& Williams, G. M. (2000). Postnatal depression myth and reality: maternal depression before and after the birth of a child. Social Psychiatry and Psychiatric Epidemiology, 19-27

Oakes, M. (2003). Perinatal Psychiatric Disorders: A Leading of Causes of Maternal Morbidity and Mortality. British Medical Bulletin, 67, 219-229

World Health Organization (WHO) (2000). Executive Board EB107/26 107 th Session 5 December Provisional agenda item 9.3 Geneva: WHO Organization.

NUTA JOURNAL, 5 (1\&2), 2074 : ISSN: 2616 - 017x 
Paykel, E. S. (2002). Mood disorders: review of current diagnostic systems. Psychopathology

Portela, A. \& Santarelli, C. (2003). Empowerment of women, men, families and communities: true partners for improving maternal and newborn health .British Medical Bulletin.

Doi:http://10.1093/bmb/ldg011

Rahman, A. et al., (2013). Interventions for common perinatal mental disorders in women in low and middle-income countries: a systematic review and meta-analysis. Bulletin World Health Organization.

Doi: http://dx.doi.org/10.2471/BLT.12.109819

Stewart, R. C. (2007). Maternal Depression and Infant Growth: A Review of Recent Evidence. Maternal Child Nutrition, 3, 94-107.

Doi: http://dx.doi.org/10.1111/j.1740-8709.2007.00088.x PMID: 17355442

Teijlingen, E. V., Simkhada, P., Devkota, V., Fanning, P., Ireland, J. Simkhada, B., Sherchan, L., Silwal, R. C., Pradhan, S., Maharjan, S. K. \& Maharjan, R. K. (2015). Mental health issues in pregnant women in Nepal. Nepal Journal of Epidemiology, 5 (3), 499-501.

United Nations (UN) (2015). The World's Women 2015 Trends and Statistics. Economic and Statistical Affairs. Department of Economic and Social Affairs. New York.

Doi: http:// 10.18356/9789210573719 e-ISBN: 978-92-1-057371-9

World Health Organization (WHO). (2004). Mental Health Promotion. Case Studies from Countries. A Joint Publiciton of the World Federation for Mental Health and the World Health Organization.

Yasamy, M. T., Maulik, P. K., Tomlinson, M., Lund, C., Ommeren, M. V. \& Saxena, S. (2011). Responsible Governance for Mental Health Research in Low Resource Countries. PLoS Med, $8(11)$.

Doi:http://10.1371/journal.pmed.1001126 e100112 